\title{
Energetics and electronic structure of semiconducting single-walled carbon nanotubes adsorbed on metal surfaces
}

\author{
Yoshiteru Takagi ${ }^{1}$ and Susumu Okada ${ }^{2}$ \\ ${ }^{1}$ Graduate School of Pure and Applied Sciences, University of Tsukuba, 1-1-1 Tennodai, Tsukuba 305-8571, Japan \\ ${ }^{2}$ Japan Science and Technology Agency, CREST, 5 Sanbancho, Chiyoda-ku, Tokyo 102-0075, Japan
}

(Received 11 April 2011; revised manuscript received 30 May 2011; published 19 July 2011)

\begin{abstract}
We investigated the electronic structure of semiconducting single-walled carbon nanotubes (CNTs) adsorbed on the (111) surfaces of $\mathrm{Au}, \mathrm{Ag}, \mathrm{Pt}$, and $\mathrm{Pd}$ and on the (0001) surfaces of $\mathrm{Mg}$ by first-principles calculations. Our calculations show that the electronic structure of the CNTs adsorbed on the metal surfaces strongly depends on the metal species. We found that on Pd surfaces, the characteristic one-dimensional electronic structure of the CNTs is totally disrupted by the strong hybridization between the $\pi$ state of the CNTs and the $d$ state of the Pd surfaces. In sharp contrast, on the Au surfaces, the CNTs retain the one-dimensional properties of their electronic structure. The distribution of the total valence charge of the CNTs on the Pd surfaces also shows a strong covalent nature between the CNTs and the surfaces. Our calculations show the importance of metal electrodes in designing CNT electronic devices.
\end{abstract}

DOI: 10.1103/PhysRevB.84.035406

PACS number(s): 73.20.At, 73.40.Ns, 61.48.De

\section{INTRODUCTION}

Miniaturization in semiconductor technology requires finding and predicting nanometer scale materials that incorporate or substitute for conventional materials in silicon-based electronic devices. Among them, carbon nanotubes (CNTs) (Refs. 1 and 2) remain important as they have various interesting electronic properties that depend on tiny differences in atomic arrangements. ${ }^{3,4}$ Their peculiar electronic properties allow for the possible fabrication of superior nanometer scale electronic devices that consist of nanotube and conventional material hybrids. For instance, it has been demonstrated that individual semiconducting nanotubes can function as field-effect transistors (FETs) (Refs. 5-16) in which nanotubes can be placed on the insulating substrates and thus form contacts with various metal surfaces such as $\mathrm{Pt}, \mathrm{Au}, \mathrm{Ca}, \mathrm{Al}$, and Pd. ${ }^{5,6}$ It has been reported that the FETs have different properties depending on the contact metal species; e.g., they exhibit $n$-type and $p$-type properties for Ca and Pd electrodes, respectively. ${ }^{13,14}$ This experimental evidence indicates that nanotubes and other conventional material hybrids are essential in these devices and they play a crucial role in determining their fundamental properties. However, little is known about the fundamental properties of the hybrid structures compared with current semiconductor technology. ${ }^{17-21}$ In particular, the stability and properties of the interface between the nanotubes and the metals are most important for the next generation of semiconductor technology.

The purpose of this work was to unravel the interplay between the tube-origin and the surface-origin electronic states in determining the stability and properties of the nanotubes attached to metal surfaces. We used single-walled carbon nanotubes (CNTs) that were adsorbed onto metal surfaces and this is considered to be a structural model of the contact between CNT and the metal electrodes. Our first-principles total energy calculation was based on density functional theory and we determined the geometric structures and properties of the nanotube-metal contact, which strongly depended on the metal species. The Fermi level of metal/CNT hybrid system is proportional to the work function of the metal species. This is in sharp contrast to conventional semiconductor-metal contacts in which the Fermi level is virtually pinned. ${ }^{22-24}$ A detailed analysis of the local density of states (LDOS) of the CNTs revealed that the LDOS on the $\mathrm{C}$ atoms at the interface region loses the characteristics of semiconducting CNTs for all the metals. At the opposite side of the CNTs for Mg, Ag, and $\mathrm{Au}$, the CNTs repair their inherent density of states (DOS) near the Fermi level. In sharp contrast, on the Pd and Pt surfaces, the LDOS of the CNTs still exhibit substantial hybridization between the CNTs and the metal surfaces.

\section{CALCULATION METHODS}

In this work, we used the TAPP (Refs. 25 and 26) code to study the geometric and electronic structures of $(10,0)$ nanotube adsorbed on metal surfaces. All the calculations were performed using density functional theory. ${ }^{27,28}$ To express the exchange-correlation energy between electrons, we used a functional form fitted to the Monte-Carlo results for a homogeneous electron gas. ${ }^{29,30}$ Ultrasoft pseudopotentials were used to describe the electron-ion interaction. ${ }^{31}$ The valence wave functions were expanded in terms of the plane-wave basis set with a cutoff energy of $30 \mathrm{Ry}$. The conjugate-gradient minimization scheme was used for the electronic structure calculation and for geometry optimization. The lattice parameters were fixed during the structural optimization. For the optimized geometry, the atoms were subjected to a force of less than 0.002 hartree/a.u. ${ }^{32}$ Integration over the two-dimensional Brillouin zone was carried out using eight $k$ points.

For this calculation, we assumed the structure shown in Fig. 1 to simulate the contact between the CNTs and the metal electrode. We chose the (111) surfaces of $\mathrm{Pd}, \mathrm{Pt}, \mathrm{Ag}$, and $\mathrm{Au}$ as substrates for CNTs. We also chose the (0001) surfaces of the $\mathrm{Mg}$ substrate. The surfaces were simulated by repeating slab models with five atomic layers. Each slab was separated by $22 \AA$ vacuum regions to determine the optimized geometries, electronic states, and energetics of the CNTs adsorbed on the surface. We therefore placed semiconducting $(10,0)$ nanotubes 
(a)

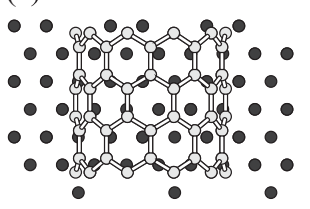

(b)
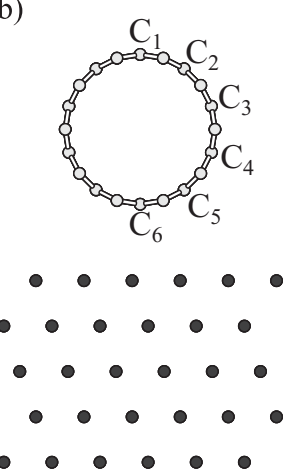

(c)

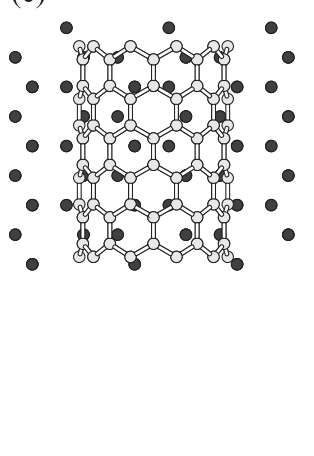

(d)
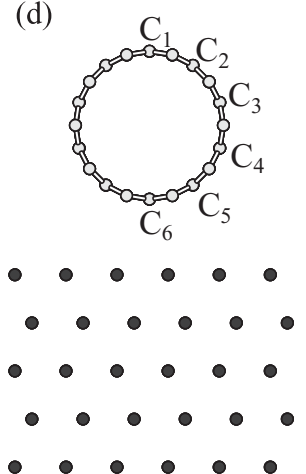

FIG. 1. Structural model of the CNTs on a metal surface. (a) Top view and (b) side view of a CNT on the (111) surface of fcc structure. (c) Top view and (d) side view of a CNT on the (0001) surface of HCP structure. The open circles and the closed circles represent carbon atoms and metal atoms, respectively. The indexes in (b) and (d) denote the atomic position in terms of the metal surface.

on the metal surfaces. Each nanotube was separated in the lateral direction by $7 \AA$ or more to simulate the characteristics of individual nanotubes on metal surfaces. We imposed a commensurability condition between the one-dimensional periodicity of the nanotube and the lateral periodicity of the metal surface. Consequently, the unit cell along the tube axis parallel to the surfaces contains double periodicity because of the zigzag nanotube. The commensurability results in the lattice constant of the nanotube on the metal surface becoming elongate by around $3 \%$.

\section{RESULTS AND DISCUSSION}

\section{A. Energetics of CNTs on the metal surfaces}

Figure 2 shows the optimized structures of the CNTs adsorbed on metal surfaces. As shown in Fig. 2(b), substantial structural relaxation was found for both the CNT and the topmost subsurface of the Pd substrate. The CNT is slightly deformed along the direction normal to the Pd surface. The $\mathrm{Pd}$ atoms at the topmost layer are shifted slightly downward. The equilibrium distance between the wall of the CNT and the Pd surfaces is $2.2 \AA$. Substantial structural relaxation was also found to occur for the CNT on the Pt surface. In this case, the CNT is elongated along the direction normal to the Pt surface. On the other hand, the topmost layer of the Pt surface is protruded, resulting in a decrease in interunit spacing. The optimum spacing between the wall of the CNTs and the surfaces was found to be $2.2 \AA$ and this is the same as for the CNTs on Pd surfaces. In sharp contrast to Pd and Pt surfaces, the CNT and the metal atoms on the substrate do not exhibit structural relaxation on $\mathrm{Au}, \mathrm{Ag}$, and $\mathrm{Mg}$ surfaces. The CNTs adsorbed on the $\mathrm{Au}, \mathrm{Ag}$, and $\mathrm{Mg}$ surfaces retain their cylindrical shape. Furthermore, these metal surfaces also retain their planar structure. In this case, the optimum distance between the walls of the CNTs and the surfaces are 2.9, 2.5, and $2.9 \AA$ for the $\mathrm{Mg}, \mathrm{Ag}$, and Au surfaces, respectively.

The local potential of the CNT/metal hybrid structure averaged along the normal to the surfaces is shown in Fig. 2. Among the metal surfaces studied, $\mathrm{Pd}$ and $\mathrm{Pt}$ seem to be the preferred electrodes for CNTs that possess good contact properties because there is no potential barrier between the CNTs and the $\mathrm{Pd} / \mathrm{Pt}$ surfaces. Therefore, the electrons are injected smoothly from the metal electrode into the CNT. These experiments show that $\mathrm{Pd}$ is a good electrode for carbon-based electronic devices. ${ }^{7,8}$ Besides the Pd surface, no potential barrier exists between the CNTs and the Ag surface. As shown later, however, the electronic structure of the CNTs on the Ag surface is very different from that of CNTs on $\mathrm{Pd} / \mathrm{Pt}$ surfaces. In sharp contrast, $\mathrm{Au}$ and $\mathrm{Mg}$ seem to be unfavorable candidates for metal electrodes which form ohmic contact with CNTs. Substantial barriers exist between the CNTs and the metal surfaces. These potential barriers may scatter the electrons that are transferred from the metal to the CNT. It should be noted that the local potential profiles in the metal region depend on the metal species. This dependency comes from the configuration of valence electrons in each metal atoms. Configurations of valence electrons are $(3 s)^{2},(4 d)^{10}$, $(4 d)^{10}(5 s)^{1},(5 d)^{9}(6 s)^{1}$, and $(5 d)^{10}(6 s)^{1}$ for $\mathrm{Mg}, \mathrm{Pd}, \mathrm{Ag}, \mathrm{Pt}$, and $\mathrm{Au}$, respectively. The local potential profile of $\mathrm{Mg}$ comprising only of $s$ orbital is the most simple and has local minimum values at atomic layers. In contrast, the local potential profile of Pd comprising only of $d$ orbital is bumpy and has some local minima due to the characteristic distribution of $d$ orbital. The local minima are not only at atomic layers but also at middle points of atomic layers. For the other metal surface possessing $d$ orbitals, the slopes of the local potential profile are more easier than that of $\mathrm{Pd}$, because of the presence of $s$ orbital.

\section{B. Electronic structure of the CNTs on the metal surfaces}

Figure 3 shows the total DOS of CNT/metal hybrid structures. Substantial DOS emerge near the Fermi level, which conceals the CNT spectrum from the total DOS. However, we can assign the first van Hove singularity of the CNTs on $\mathrm{Mg}, \mathrm{Ag}$, and $\mathrm{Au}$, by comparing with LDOS for these systems. As will be mentioned later, the $E_{11}$ gap clearly exits in LDOS for the CNTs on $\mathrm{Mg}, \mathrm{Ag}$, and $\mathrm{Au}$. It is clear that electrons are injected into the CNTs from the Mg surface since the lowest unoccupied state of the CNT is located below the Fermi level. In sharp contrast, for the Ag and Au surfaces, a charge transfer does not occur. On the Ag surfaces, the Fermi level is located just below the lowest unoccupied state of the CNTs. These facts indicate that the CNTs on the Ag surfaces can potentially be an $n$-type channel as for the $\mathrm{Mg}$ electrode. On the Au surfaces, the Fermi level is located just above the 
(a)

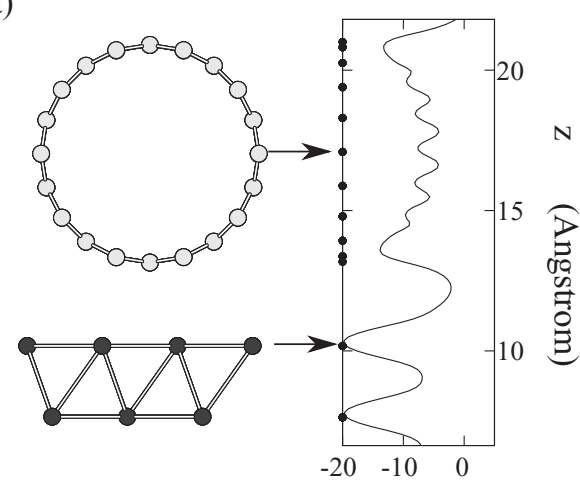

av. $\operatorname{Vlocal}(\mathrm{z})$

$(\mathrm{eV})$

(c)

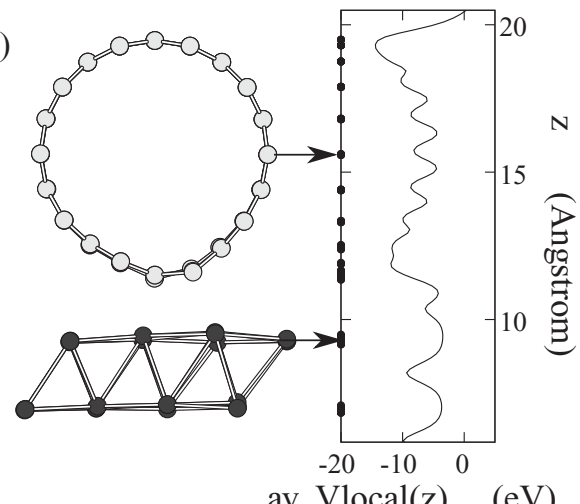

av. $\operatorname{Vlocal}(\mathrm{z}) \quad(\mathrm{eV})$ (b)

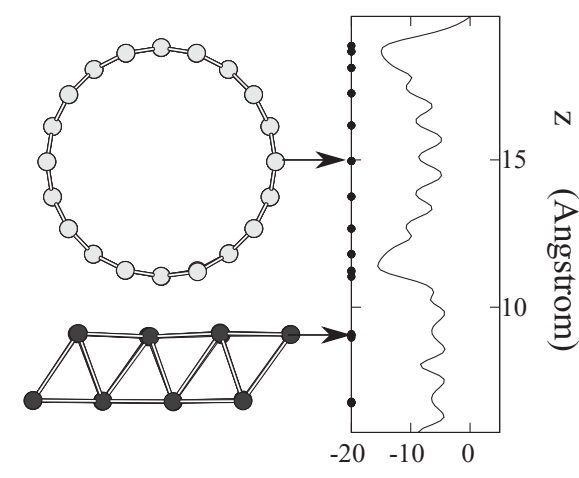

av. $\operatorname{Vlocal}(\mathrm{z})$

$(\mathrm{eV})$

(d)

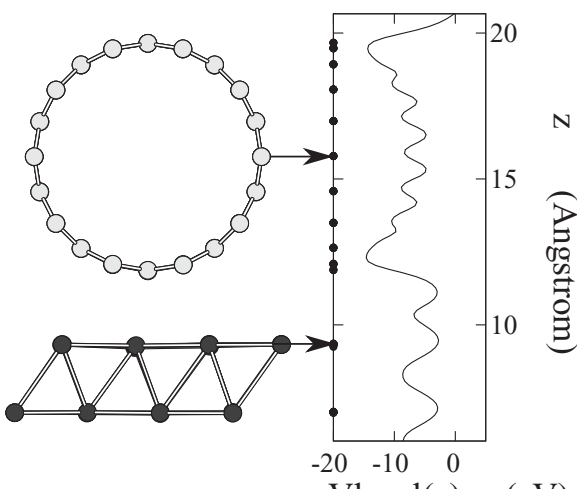

av. $\operatorname{Vlocal}(\mathrm{z})$

(e)

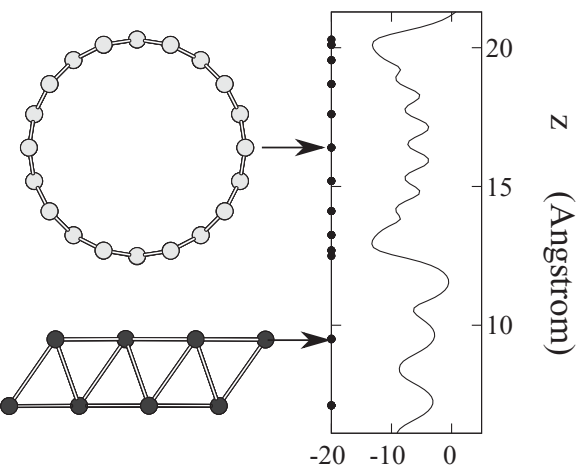

av. $\operatorname{Vlocal}(z)$

$(\mathrm{eV})$

FIG. 2. Optimized structure (left panel) and plane-averaged local potential (right panel) for the CNTs on (a) Mg, (b) Pd, (c) Pt, (d) Ag, and (e) Au substrates. In the left panels, the open circles and the closed circles represent carbon atoms and metal atoms, respectively. In the right panels, the black dots represent the position of carbon atoms or metal atoms. Equilibrium distance separation between the wall of the CNT and the $\mathrm{Mg}, \mathrm{Pd}, \mathrm{Pt}, \mathrm{Ag}$, and Au surfaces is $2.9,2.2,2.2,2.5$, and $2.9 \AA$, respectively.

highest occupied state of the CNT. Therefore, the CNTs with Au electrodes may exhibit a $p$-type character. In sharp contrast to the $\mathrm{Ag}, \mathrm{Au}$, and $\mathrm{Mg}$ surfaces, it is difficult to assign the peaks in the spectra that originate from the CNTs on the Pt and Pd surfaces. This fact indicates that the $\pi$ states of the CNT are substantially hybridized with the $d$ states of surface Pd and $\mathrm{Pt}$ atoms. It is thus important to investigate how the local electronic structure of a CNT is modulated by the adsorption of CNTs onto the metal surfaces. In particular, it is important to determine if the semiconducting CNTs on the metal surfaces retain their semiconducting properties on the surfaces. To obtain theoretical insight into this issue, we investigated the LDOS on each $\mathrm{C}$ atom along the circumference of a CNT.

Figures 4, 5, and 6 show the LDOS of the CNTs adsorbed on the $\mathrm{Mg}$ surface, the $\mathrm{Ag}$ surface, and the $\mathrm{Au}$ surface, respectively. As shown in these figures, all the $\mathrm{C}$ atoms in the CNTs retain the characteristic nature of a CNT. For each atomic site, the peaks that originate from the first van Hove singularities are present in the spectra. In particular, at the atomic sites facing the vacuum region, the two peaks associated 


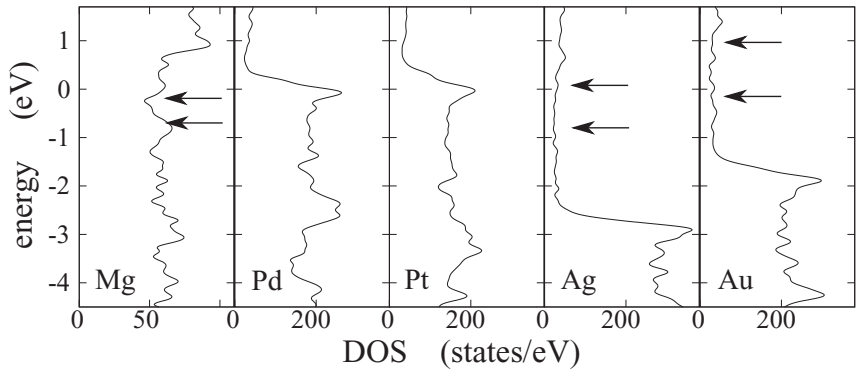

FIG. 3. Density of states for the CNTs on Mg, Pd, Pt, Ag, and Au substrates. The Fermi level is at $0 \mathrm{eV}$. The arrows represent the $E_{11}$ band gap of the CNTs, respectively.

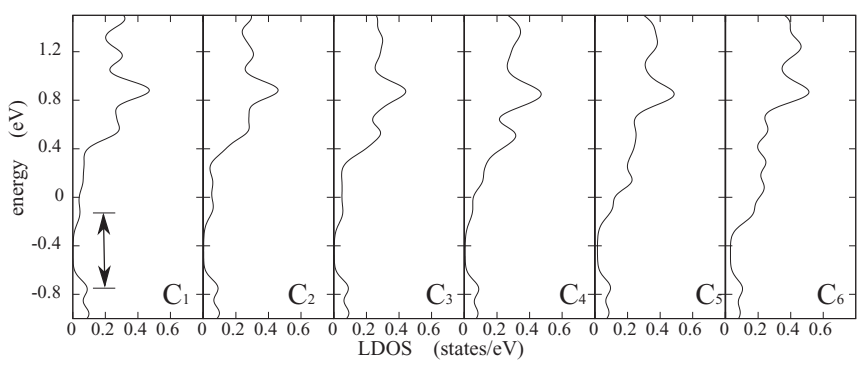

FIG. 4. Local density of states (LDOS) around $\mathrm{C}_{1}, \mathrm{C}_{2}, \mathrm{C}_{3}, \mathrm{C}_{4}$, $\mathrm{C}_{5}$, and $\mathrm{C}_{6}$, which are shown in Fig. 1 , for the CNTs on a Mg (0001) surface. The Fermi energy is at $0 \mathrm{eV}$. The arrow represents the $E_{11}$ band gap of the CNTs, respectively.

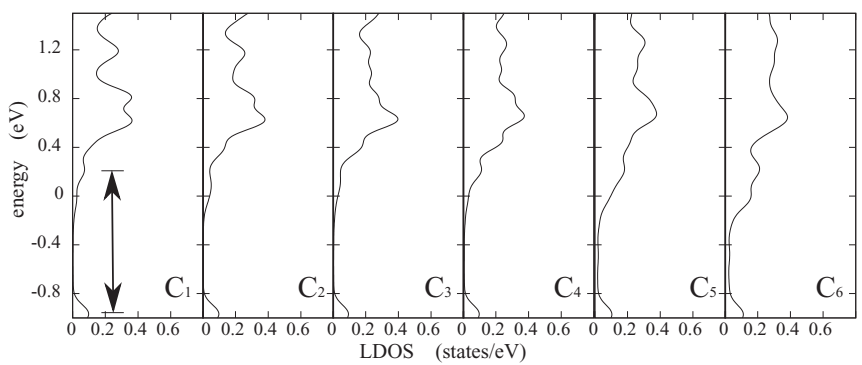

FIG. 5. Local density of states (LDOS) around $\mathrm{C}_{1}, \mathrm{C}_{2}, \mathrm{C}_{3}, \mathrm{C}_{4}$, $\mathrm{C}_{5}$, and $\mathrm{C}_{6}$, which are shown in Fig. 1 , for the CNTs on a $\mathrm{Ag}(111)$ surface. The Fermi energy is at $0 \mathrm{eV}$. The arrow represents the $E_{11}$ band gap of the CNTs, respectively.

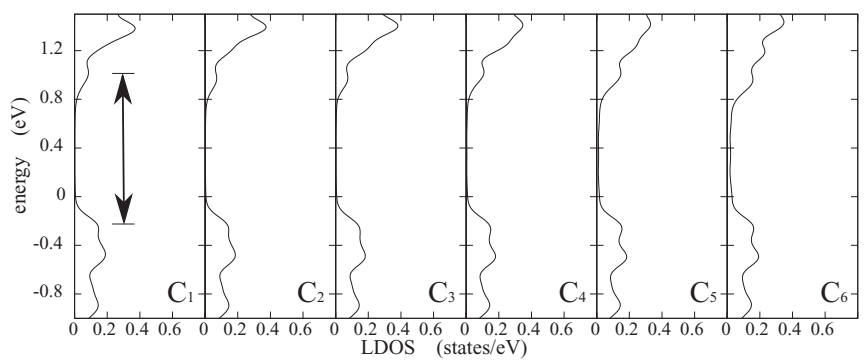

FIG. 6. Local density of states (LDOS) around $\mathrm{C}_{1}, \mathrm{C}_{2}, \mathrm{C}_{3}, \mathrm{C}_{4}$, $\mathrm{C}_{5}$, and $\mathrm{C}_{6}$, which are shown in Fig. 1, for the CNTs on an Au (111) surface. The Fermi energy is at $0 \mathrm{eV}$. The arrow represents the $E_{11}$ band gap of the CNTs, respectively.

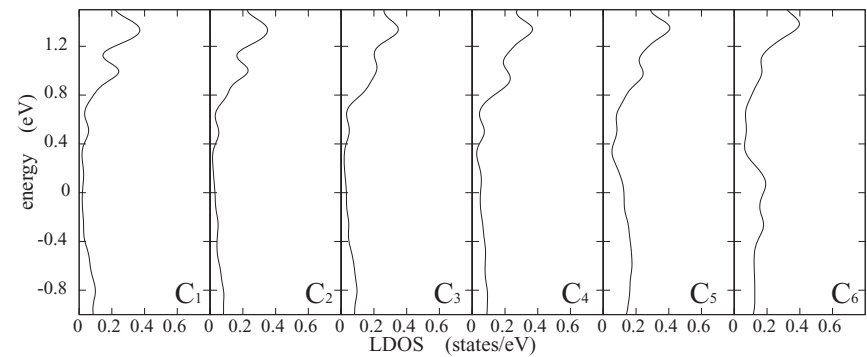

FIG. 7. Local density of states (LDOS) around $\mathrm{C}_{1}, \mathrm{C}_{2}, \mathrm{C}_{3}, \mathrm{C}_{4}$, $\mathrm{C}_{5}$, and $\mathrm{C}_{6}$, which are shown in Fig. 1 , for the CNTs on a Pd (111) surface. The Fermi energy is at $0 \mathrm{eV}$.

with the $E_{11}$ gap are clear in the spectra and the LDOS are absent between these two peaks. Upon approaching the metal surfaces, metal-induced gap states appear ${ }^{23,24}$ and give small but substantial DOS in the band gap of the CNTs. It should be noted that the peak position weakly depends on the atomic site. This shift should correspond with band bending in the semiconductor at the interfaces with the metal surfaces. The CNTs on the $\mathrm{Ag}, \mathrm{Au}$, and $\mathrm{Mg}$ surfaces mostly retain their electronic structures except for the above-mentioned differences.

In contrast to the $\mathrm{Ag}, \mathrm{Au}$, and $\mathrm{Mg}$ surfaces, the electronic structure of the CNTs is drastically modulated upon their adsorption onto Pd and Pt surfaces. The LDOS of a CNT on Pd and Pt surfaces is shown in Figs. 7 and 8, respectively. As shown in these figures, the characteristic feature of the DOS of CNT is absent and a finite DOS emerges around the Fermi level. For the $\mathrm{C}_{6}$ atoms facing the $\mathrm{Pd} / \mathrm{Pt}$ surfaces, a large DOS without significant structure emerges at the Fermi level. Furthermore, a small but finite DOS emerges around the Fermi level even on $\mathrm{C}$ atoms facing the vacuum region. This modulation is due to strong hybridization between the $\pi$ state of the $\mathrm{C}$ atoms and the $d$ states of the Pd/Pt surfaces. The LDOS of the CNTs on the Pd/Pt surfaces indicates that the CNTs lose their original character, at least in the nanometer range.

Finally, we focused on the Fermi level of our CNT/metal hybrid system as a function of the work function of the metal species. It is well known that the Fermi level of conventional semiconducting materials such as $\mathrm{Si}$ and GaAs is pinned at a certain energy. However, the Fermi level is not pinned in the CNT/metal hybrid system. As shown in Fig. 9, the Fermi level $E_{f}$ of the CNT/metal hybrid system measured from the vacuum level is almost proportional to the work function of the metal species. For CNTs on Mg whose work function is the

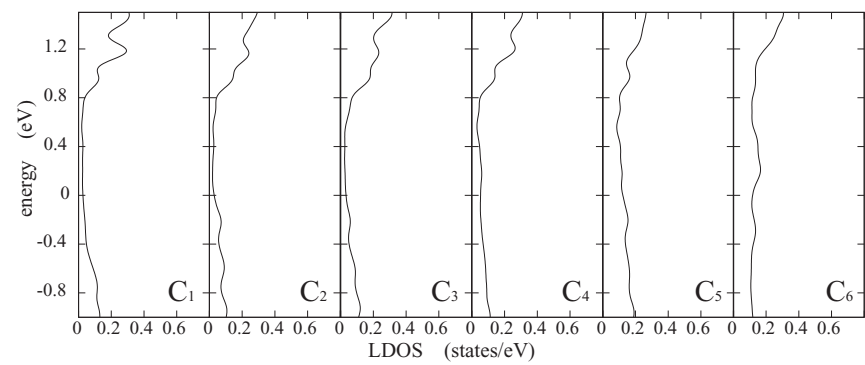

FIG. 8. Local density of states (LDOS) around $\mathrm{C}_{1}, \mathrm{C}_{2}, \mathrm{C}_{3}, \mathrm{C}_{4}$, $\mathrm{C}_{5}$, and $\mathrm{C}_{6}$, which are shown in Fig. 1 , for the CNTs on a Pt (111) surface. The Fermi energy is at $0 \mathrm{eV}$. 


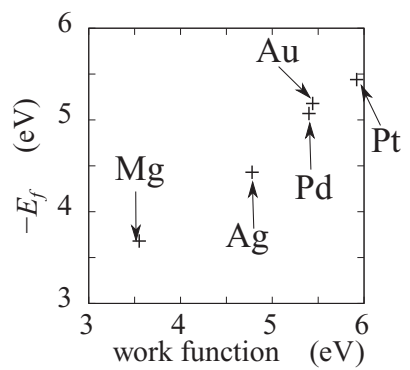

FIG. 9. Fermi level of CNT/metal system as a function of the work function of the metal substrate. $E_{f}$ is the Fermi level measured from the vacuum level. Here, the work functions of the metal species are calculated values.

smallest among the metals studied here, $-E_{f}$ is the shallowest. With increasing the work function, $-E_{f}$ also monotonically increases. Therefore, the Fermi level of the CNT/metal hybrid system strongly depends on the work function of metal surface. The fact indicates the possibility that the height of the Schottky barrier between CNT and metal surface is tunable by selecting metal species. This agrees well with experimental results, which showed that CNTs can function as $p$-type and $n$-type channels depending on the metal electrode species. ${ }^{14}$

\section{CONCLUSION}

We investigated the electronic structures of CNTs on (111) surfaces of $\mathrm{Ag}, \mathrm{Au}, \mathrm{Pd}$, and $\mathrm{Pt}$, and the (0001) surfaces of $\mathrm{Mg}$ by performing first-principles total-energy calculations. Our calculations show that the electronic structure of the CNTs adsorbed on metal surfaces strongly depends on the metal species. For $\mathrm{Ag}, \mathrm{Au}$, and $\mathrm{Mg}$ surfaces, the CNTs mostly retain their pristine electronic structures upon adsorption. In particular, for the $\mathrm{C}$ atoms far from the metal surfaces, the characteristic DOS for the CNTs are found to emerge around the Fermi level. On the other hand, for the $\mathrm{C}$ atoms that face the metal surfaces, metal-induced gap states appear and give small but substantial DOS in the band gap of the CNTs. In sharp contrast, the characteristic electronic feature of the CNTs is totally disrupted after adsorption onto Pd and Pt surfaces due to the hybridization between the $\pi$ state of the CNTs and the $d$ orbital of the metal surfaces. Our analysis of the total valence charge clearly shows that CNTs form covalent bonds with the $\mathrm{Pd}$ surface and the Pt surface. Finally, our calculations indicate that the metal-induced gap states and the interface states caused by hybridization between the $\pi$ state of the CNTs and the $d$ orbital of the metal surfaces do not pin the Fermi level in the $\mathrm{CNT} /$ metal hybrid system. The Fermi level at the CNT/metal interface is proportional to the magnitude of the work function of the metal species.

\section{ACKNOWLEDGMENTS}

This work was supported by CREST, Japan Science and Technology Agency, and a Grant-in-Aid for Scientific Research from the Ministry of Education, Culture, Sports, Science and Technology of Japan. Computations were performed on an NEC SX-8/4B at the University of Tsukuba, on an NEC SX-9 at the Institute for Solid State Physics, The University of Tokyo, on an NEC SX-9 at the Information Synergy Center, Tohoku University, and on an NEC SX-9 at the Cybermedia Center, Osaka University.
${ }^{1}$ S. Iijima, Nature 354, 56 (1991).

${ }^{2}$ M. S. Dresselhaus, G. Dresselhaus, and P. C. Eklund, Science of Fullerenes and Carbon Nanotubes (Academic Press, San Diego, 1996).

${ }^{3}$ N. Hamada, S. I. Sawada, and A. Oshiyama, Phys. Rev. Lett. 68, 1579 (1992).

${ }^{4}$ R. Saito, M. Fujita, M. S. Dresselhaus, and G. Dresselhaus, Appl. Phys. Lett. 60, 2204 (1992).

${ }^{5}$ S. J. Tans, A. R. M. Verschueren, and C. Dekker, Nature 393, 49 (1998).

${ }^{6}$ R. Martel, T. Schmidt, H. R. Shea, T. Hartel, and Ph. Avouris, Appl. Phys. Lett. 73, 2447 (1998).

${ }^{7}$ D. Mann, A. Javey, J. Kong, Q. Wang, and H. Dai, Nano Lett. 3, 1541 (2003).

${ }^{8}$ A. Javey, J. Guo, Q. Wang, M. Lundstrom, and H. Dai, Nature 424, 654 (2003).

${ }^{9}$ A. Javey, J. Guo, D. B. Farmer, Q. Wang, R. G. Gordon, M. Lundstrom, and H. Dai, Nano Lett. 4, 447 (2004).

${ }^{10}$ M. H. Yang, K. B. K. Teo, W. I. Milne, and D. G. Hasko, Appl. Phys. Lett. 87, 253116 (2005).

${ }^{11}$ R. V. Seidel, A. P. Graham, J. Kretz, B. Rajasekharan, G. S. Duesberg, M. Liebau, E. Unger, F. Kreupl, and W. Hoenlein, Nano Lett. 5, 147 (2005).

${ }^{12}$ Z. Chen, J. Appenzeller, J. Knoch, Y. Lin, and Ph. Avouris, Nano Lett. 5, 1497 (2005).

${ }^{13}$ Y. Nosho, Y. Ohno, S. Kishimoto, and T. Mizutani, Appl. Phys. Lett. 86, 073105 (2006).
${ }^{14}$ Y. Nosho, Y. Ohno, S. Kishimoto, and T. Mizutani, Nanotechnology 17, 3412 (2006).

${ }^{15} \mathrm{X}$. Song, X. Han, Q. Fu, J. Xu, N. Wang, and D. P. Yu, Nanotechnology 20, 195202 (2009).

${ }^{16}$ A. D. Franklin and Z. Chen, Nat. Nanotechnol. 5, 858 (2010).

${ }^{17}$ F. Léonard and J. Tersoff, Phys. Rev. Lett. 84, 4693 (2000).

${ }^{18}$ B. Shan and K. Cho, Phys. Rev. B 70, 233405 (2004).

${ }^{19}$ S. Okada and A. Oshiyama, Phys. Rev. Lett. 95, 206804 (2005).

${ }^{20}$ T. Meng, C. Wang, and S. Wang, J. Appl. Phys. 102, 013709 (2007).

${ }^{21}$ Y. Matsuda, W. Deng, and W. A. Goddard III, J. Phys. Chem. C 111, 11113 (2007).

${ }^{22}$ A. M. Cowley and S. M. Sze, J. Appl. Phys. 36, 3212 (1965).

${ }^{23}$ V. Heine, Phys. Rev. 138, A1689 (1965).

${ }^{24}$ S. G. Louie and M. L. Cohen, Phys. Rev. B 13, 2461 (1976).

${ }^{25}$ M. Tsukada, Computer Program Package TAPP (University of Tokyo, Tokyo, Japan, 1983-2010).

${ }^{26}$ J. Yamauchi, M. Tsukada, S. Watanabe, and O. Sugino, Phys. Rev. B 54, 5586 (1996).

${ }^{27}$ P. Hohenberg and W. Kohn, Phys. Rev. 136, B864 (1964).

${ }^{28}$ W. Kohn and L. J. Sham, Phys. Rev. 140, A1133 (1965).

${ }^{29}$ J. P. Perdew and A. Zunger, Phys. Rev. B 23, 5048 (1981).

${ }^{30}$ D. M. Ceperley and B. J. Alder, Phys. Rev. Lett. 45, 566 (1980).

${ }^{31}$ D. Vanderbilt, Phys. Rev. B 41, 7892 (1990).

${ }^{32}$ O. Sugino and A. Oshiyama, Phys. Rev. Lett. 68, 1858 (1992). 